\section{ALLEVIATION OF INTRACTABLE PAIN*}

BY

RAYMOND GAVIN, F.F.A. R.C.S., D.A.

Consultant Anctesthetist, Taunton and South Somerset Group of Hospitals

The past five years have seen the development of a new and more positive approach to pain to which the adjective "intractable" is in consequence becoming less applicable, although this description is still true of many cases of incurable cancer and non-malignant diseases, such as arthritis, which cause so much distress to the aged.

Much of the renewed interest in this condition has perhaps been due to the development of new analgesic drugs and trancuillizers that continue to appear in increasing volume, and to the work of Maher (1955, 1957), Nathan and Scott (1958), and Brown (1958) on intrathecal phenol.

No consideration of the problem of pain is possible without the appreciation that this is an intensely personal symptom and that pain thresholds vary widely in individuals. Congenital factors may play a part here, although, as Russell (1959) points out, this does not materially aflect the problem except in so far as emotional factors may lower thresholds and complicate the pattern, pushing it further into the field of psychotherapy.

It is natural that the challenge of intractable pain should be accepted by anaesthetists, for they are well equipped to make a significant contribution to its treatment, both by virtue of a wide knowledge of modern analgesic drugs and by their experience of the approach to the subarachnoid space, which they have gained from the practice of spinal analgesia.

\section{Analgesic Drugs}

The use of pain-killing drugs has for centuries past been the front line of attack against the problem of pain. Because of their ease of administration and undeniable reliability, it is certain that they will continue to occupy this position. Morphine and its derivatives have long held pride of place here-so much so that they come to mind almost reflexly when one is faced with the treatment of these patients. Nevertheless, morphine has many undesirable side-effects, of which nausea, vomiting, constipation, and the clouding of mental processes are well known. At times the hypnotic effect of morphine is highly desirable, but probably mostly in the terminal stages of pain from malignant disease. Perhaps far too many patients are submitted too early to the merciful but despairing relief afforded by morphine, and the last months of life are spent in disorientation and uselessness, which is profoundly disturbing to their family and friends.

In the earlier stages of pain for which there is no medical or surgical cure, much can be done by the judicious administration of mild analgesic drugs; of these, tab. codein. co. is representative, and its longcontinued use does not bring unwanted side-effects in its train.

In recent years there has been a considerable increase in the phvsician's armamentarium whereby he may

* Read in the Section of Anaesthetics at the Annual Meeting of the British Medical Association, Torquay, 1960. control the more severe pain without recourse to his old ally, morphine. Many of these drugs are specific analgesics and are almost without hypnotic action. Under their influence patients may be relieved of their pain while fully able to work or deal with business affairs. Among those drugs which we might call the potent analgesics the following have shown considerable promise in the control of intractable pain: oxvcodone pectinate, dihydrocodeine bitartrate, levorphanol tartrate, methadone hydrochloride, and dextromoramide.

Oxycodone Pectinate.-This compound consists of dihydrohydroxycodeinone combined with the pectin macro-molecule. The active alkaloid is released slowly in the body and when given intramuscularly in doses of 10-20 mg. its duration of action may last from 8 to 14 hours. Belam (1957), reporting on the use of this drug in 100 patients, did not find any sedative action, but confirmed its long-acting properties. I have administered this preparation in doses of $10-15 \mathrm{mg}$. during operations where a painful convalescence can be expected-for example, haemorrhoidectomy-and have been impressed with the degree and duration of the analgesic effect. In intractable pain controlled by morphine it can adequately replace the latter drug, although dosage may have to be initially as high as $30 \mathrm{mg}$. six- or eight-hourly. It has recently become available in $30-\mathrm{mg}$. suppositories and can therefore be self-administered by ambulant patients.

Dihydrocodeine Tartrate.-Gravenstein et al. (1956) reported this drug to be almost as effective as morphine with fewer side-effects. It is active when administered orally (30-60 mg.) or parenterally $(30-50 \mathrm{mg}$.) and has no hypnotic or sedative properties.

Levorphanol Tartrate.-This drug was synthesized in 1949. Of two isomers, the dextro-isomer has a selective action on the cough centre. The laevo-isomer has a powerful analgesic action with few side-effects. It is reliable when taken by mouth in tablets of $1.5 \mathrm{mg}$., and up to three may be given to patients with severe pain. It is available in ampoules of $2 \mathrm{mg}$. for parenteral administration, and this should produce an effect for six to eight hours.

Methadone Hydrochloride.-This drug probably acts directly on the thalamus and, unlike morphine, does not cause depression of the higher centres. It is about equipotent with morphine in analgesic action and has a depressant action on the cough centre.

Dextromoramide.-This new and very potent analgesic is 7 to 18 times as powerful as morphine. It is one of the very few which are equally as effective $u$ hether administered by the oral or the parenteral route, and its therapeutic effect is very rapid. Duration of action depends on dosage, but averages four hours when a normal dose of $5-10 \mathrm{mg}$. is taken orally. Intravenously, 1-2 mg. produces effects which are observed almost immediately, and potentiation of other hypnotic drugs, concurrently administered, may be marked. Nausea, vomiting, and drowsiness are occasionally seen, but infrequently compared with morphine, and constipation has not been observed.

\section{Addiction to Analgesic Drugs}

It must be assumed that all the potent analgesics are capable of giving rise to addiction. Nevertheless, I have the very strong impression that analgesic drugs which have little or no hypnotic properties are very slow to 
produce addiction when compared with drugs which have this action in marked degree.

Although pain can often be adequately controlled by the use of analgesic drugs, prolonged administration often tends to produce depression of mental activity, and much benefit can be derived by the use of amphetamine or methyl phenidate hydrochloride to elevate mood. The tranquillizing action of the phenothiazine group can also be useful here, although the hypnotic action of analgesics, even if minimal, is often thus potentiated, and care is needed in balancing the dosage accordingly. Prochlorperazine was found by Bowen (1959) to be superior to chlorpromazine in controlling nausea and vomiting while showing only very weak potentiating properties.

\section{Nerve Conduction Blockade}

Apart from treatment by drugs, pain can be abolished by blocking the pathways by which pain reaches the central nervous system. The detailed consideration of surgical operations for the relief of severe pain are out of place here, belonging as they do within the sphere of neurosurgery. Nevertheless, they illustrate very well the degree of selectivity, in regard to pain fibres, which must be the basis of all attempts to combat pain, and they are therefore worthy of brief mention.

Interruption of pain conductivity can be brought about by (1) division of peripheral nerves, (2) section of posterior roots (post-rhizotomy), and (3) cordotomy, or diversion of tracts within the cord itself.

Most peripheral nerves contain both motor and sensory fibres and can regenerate after division. Clearly, therefore, the first of these procedures must be classified as crude and undesirable. At posterior root level, however, the afferent pathways may be cut without influencing motor function, but the loss of proprioceptive sensation and touch tends to trophic changes and may render a limb useless and a burden to its owner. The operation of cordotomy is a much more refined approach, directed as it is to the division of specific tracts conducting pain and temperature after dissociation within the cord, and leads to no more unpleasant sensations than mild paraesthesiae.

\section{Technique}

The technique, first described by Maher (1955), attempts to achieve "selective chemical rhizotomy" by the intrathecal injection of phenol in heavy conveying solutions and is of considerable interest, especially in the control of intractable pain in malignant disease.

This procedure may require some degree of mental reorientation on the part of anaesthetists, conditioned as they are by the necessity, heretofore, of excluding substances such as phenol from gaining access to the C.S.F. However, the corrosive action of phenol does not appear in the anhydrous state, and, whilst it is soluble in glycerin and ethyl iodophenylundecylate (" myodil "), it is rapidly absorbed by nervous tissue when brought into contact with nerve roots and ganglia in this state. This solution possesses, in addition, self-sterilizing properties, although it is usual to prepare fresh solutions sterilized by dry heat. Dogliotti (1931) used the intrathecal route to administer alcohol, in an attempt to interrupt pain-conduction in nerve roots without loss of sensory or motor function. In the C.S.F., alcohol is rapidly diluted before it damages the more myelinated motor roots, but not before producing a degree of damage to the fibres conducting pain. Accurate position- ing of drugs injected below the arachnoid is essential, and alcohol, being exceedingly hypobaric, is not always easy to control.

A $5 \%$ solution of phenol in glycerin is heavier than C.S.F. and easier to position within the spinal canal. Moreover, it is not so susceptible to minor inaccuracies of spinal tilt. A solution of this strength will affect permanently the small pain fibres or cells of the posterior root ganglion without producing unwanted neurological sequelae.

Intrathecal phenol is especially valuable in severe pain from malignant deposits arising from primary growths of the leg-for example, sarcoma-or pelvis. Subarachnoid puncture is easier below D 12 and within the lumbar stretch, and there is no danger here of cord damage. With the use of a downward slope towards the sacrum, the solution can be made to run over the affected roots while the patient lies on the painful side. $1 \mathrm{ml}$. of solution is usually sufficient to influence four or five roots, and repeated injections can be given to cover all affected parts. Relief from pain is obtained within a few minutes if positioning has been accurate. and failures have been ascribed to the invasion of the posterior root ganglion by malignant cells and consequent sheltering of the nerve elements (Maher, 1957). Unilateral attack is less likely to cause bladder disturbances, which, however, are usually transitory and manifest by retention or slight hypotonia for 24 hours Pre-existing bladder dysfunction, however, may be rendered permanently worse if the lower sacral roots are involved in the injection.

In the cervical region rather better results have been obtained by subdural than by subarachnoid injections of phenol in myodil (Maher, 1959, personal communication). The approach to the cervical subarachnoid space has been described as easily performed, although this has not been so easy in my own limited experience. Pressure of C.S.F. in this region, with a patient in the sitting position, is low, and, using a needle of 21 gauge, which is desirable, considerable patience on the part of the operator (and patient) is necessary to be sure the subarachnoid space has been reached. If the needlepoint is carefully withdrawn until C.S.F. just ceases to flow, injection of 1 to $1.5 \mathrm{ml}$. may be made subdurally to affect cervical roots in brachial pain and, when myodil is the solvent, radiological confirmation of correct positioning may be obtained.

If injection is made during the withdrawal of the needle-point rather than at the position of maximal advance the extensive damage which would result from injection of phenol solutions into the cord substance is obviated, whilst the danger of damage to the cord by the dry needle-point has probably been overestimated.

The choice between glycerin and myodil as a solvent for phenol is a matter of individual preference. If radiological evidence is desirable to confirm accurate positioning then myodil will be selected. However, there is evidence that myodil is very slow to disappear from the site of deposition and tends to form a sludgy deposit around the nerve roots which may render subsequent injections ineffective; therefore, if repeated instillations are probable, glycerin may be preferable. Myodil, however, is less viscous and easier to inject through a fine needle than glycerin, which should be warmed before injection.

Phenol dissolved in glycerin is considered by Brown (1958) to be more potent owing to the phenol being 
more readily released into the C.S.F., and he therefore recommends that its use be restricted to the lowest sacral roots. If, however, this reasoning is correct, it is difficult to understand why, since the concentration is more likely to occur towards the tip of the dural sac, the increased potency might not be expected to produce bladder disturbances by involvement of the second, third, and fourth sacral roots.

The action of phenol is selective in that it causes a permanent destruction of the smallest nerve fibres and cells of the posterior root ganglion while leaving the larger fibres comparatively unaffected. Although severe exacerbations of pain, due to some concomitant extension of the disease, such as spontaneous fracture of femoral heads, may penetrate the curtain of analgesia, such "incident" pain is of short duration and can be adequately controlled by drugs.

Experiences with intrathecal phenol have led Maher (1960) to describe two pathways by which pain reaches the central nervous system. The larger fibres, possessing a high-conduction rate and requiring a brisk stimulus, may be responsible for pain of sudden onset, whilst the small non-medullated fibres are responsible for the conduction, much more slowly, of continuous pain. The larger fibres transmit pain quickly to elicit defensive reflexes and may be concerned in incident pain, but it is the smaller fibres which are most susceptible to the action of phenol in the concentrations most commonly used intrathecally.

The causes of failure of phenol to produce analgesia of nerve roots may be due to fibrosis or invasion of nerve elements by tumour cells; radiculitis, giving rise to inflammatory swelling of nerve roots which tend to exclude phenol by filling the root pockets; as well as by the use of inadequate concentrations of phenol or inaccurate posturing. In cases resistant to $5 \%$ phenol, Maher obtained success by the addition of small doses of silver nitrate (1/80 gr. (0.8 mg.) in $1 \mathrm{ml}$.) and found that this addition could also make permanent a temporary phenol effect at the expense of slight meningeal reactions.

Intrathecal phenol, in concentrations higher than $5 \%$, has been used successfully (Nathan, 1959) to abolish the spasticity of paraplegics by destroying the anterior roots. Phenol $10-20 \%$ in glycerin is required, but some effects on the posterior roots are inescapable and some loss of sensation is inevitable. Nevertheless, the conversion of spastic to flaccid paralysis and the abolition of spasms in these unfortunate cases can thus be achieved without an operation and renders life much more bearable and nursing easier.

\section{Pain-relief Clinics}

"Light is the task where many share the toil." - HOMER.

In conclusion, let us spare a thought with regard to the establishment of clinics specifically for the treatment of this most insistent of man's afflictions. While we may classify pain clinically into visceral, somatic, or psychogenic compartments, only rarely does the pain fail to affect the psyche, and the psyche the pain.

There is little doubt that, whatever methods one employs to combat intractable pain, the earlier treatment is begun the more effective is it likely to be, and the existence of clinics specifically for this purpose leads to the creation of confidence between patient and doctor which is all-important to success.
Those centres which now operate in Britain are all too few, but their establishment is indicative of an increasing interest among doctors to meet the challenge which severe chronic pain offers.

Anaesthetists have a vital part to play in this sphere ; indeed, many of the clinics at present operating owe their existence to the extension of clinical anaesthesia into therapeutic fields. In some the accent may be on nerve-blocking and physiotherapy, in others on drug therapy or the neurosurgical approach, but even the psychotherapist or hypnotherapist cannot be excluded from this field. Such clinics cannot develop as they should without the skill and advice which only a panel of correlated specialists can supply and which is so necessary to meet, and combat, an enemy of such magnitude and resilience.

\section{Summary}

The treatment of severe chronic pain merits a new appraisal in the light of recent developments in analgesic drugs and methods of achieving selective action on nerve roots by subarachnoid injections.

The complexity of factors influencing appreciation of pain must be accepted by workers in this field.

The use of morphine might well be delayed or even relegated to the terminal stages of pain in malignant disease.

In the severe pain of primary or secondary cancer, subdural phenol in $5 \%$ concentration in lipoid solvents has proved a relatively safe, and sometimes remarkably efficient, therapeutic agent, especially in pelvic and lower-limb growths. The experience gained by anaesthetists of the gravitational control of solutions injected below the arachnoid can be utilized to advantage in this form of therapy.

Life is almost certainly prolonged where the pain of malignant disease is adequately controlled, and this must inevitably rob cancer of much of the dread associated with it.

Pain-relief clinics, successfully operating in some centres, are essential for the early and complete treatment of severe pain.

I wish to thank my medical and surgical colleagues of the Taunton and Somerset and Bridgwater Hospitals for kindly referring cases to me, and Dr. Robert Maher for his unstinting encouragement and advice.

\section{REFERENCES}

Belam, O. H. (1957). Brit. J. Anaesth., 29, 190.

Bowen, E. H. (1959). Anesth Anal. Curr. Res., 38, 14.

Brown, A S. (1958). Lancet, 2, 975.

Dogliotti, A. M. (1931). Rev. neurol., 2, 485.

Gravenstein, J. S., Smith, G. M., Sphire, R. D., Isaacs, J. P. and Beecher, H. K. (1956). New Engl. J. Med., 254, 877. Maher, R. M. (1955) Lancet, 1. 18.

- (1957). Ibid., 1, 16. - (1960). Ibid., 1, 895 .

Nathan, P. W. (1959). Ibid., 2, 1099.

Nathan, P. W. (1959). (1958). Ibid., i, 76

Russell, W. R. (1959). Proc. roy. Soc. Med., 52, 983.

A new American journal, DSH Abstracts, is being published by Deafness Speech and Hearing Publications, and contains abstracts of literature in the areas of deafness, speech, and hearing. It will be published quarterly in January, April, July, and October. Publication was made possible by a training grant provided by the Office of Vocational Rehabilitation, Department of Health, Education, and Welfare. Inquiries should be sent to the Business Manager, Deafness Speech and Hearing Publications. American Speech and Hearing Association, 1001 Connecticut Avenue, N.W., Washington 6, D.C. 